\title{
Quantitative terahertz emission nanoscopy with multiresonant near-field probes
}

\author{
Fabian Mooshammer, ${ }^{1,2, *}$ (1) Markus Plankl, ${ }^{1}$ Thomas Siday, ${ }^{1}$ Martin Zizlsperger, ${ }^{1}$ \\ Fabian Sandner, ${ }^{1}$ Rocco Vitalone, ${ }^{2}$ Ran Jing, ${ }^{2}$ Markus A. Huber, ${ }^{1}$ D. N. Basov, ${ }^{2}$ and \\ RUPERT HUBER ${ }^{1}$ \\ ${ }^{1}$ Department of Physics and Regensburg Center for Ultrafast Nanoscopy (RUN), University of Regensburg, 93040 Regensburg, Germany
${ }^{2}$ Department of Physics, Columbia University, New York, New York 10027, USA \\ *Corresponding author: fabian.mooshammer@ur.de
}

Received 6 May 2021; revised 25 June 2021; accepted 28 June 2021; posted 28 June 2021 (Doc. ID 430400 ); published 22 July 2021

By sampling terahertz waveforms emitted from InAs surfaces, we reveal how the entire, realistic geometry of typical near-field probes drastically impacts the broadband electromagnetic fields. In the time domain, these modifications manifest as a shift in the carrier-envelope phase and emergence of a replica pulse with a time delay dictated by the length of the cantilever. This interpretation is fully corroborated by quantitative simulations of terahertz emission nanoscopy based on the finite element method. Our approach provides a solid theoretical framework for quantitative nanospectroscopy and sets the stage for a reliable description of subcycle, near-field microscopy at terahertz frequencies. () 2021 Optical Society of America

https://doi.org/10.1364/OL.430400

Ultrafast currents in solids spontaneously emit electromagnetic radiation, whose frequency often lies in the elusive terahertz $(\mathrm{THz})$ regime [1,2] owing to the prevalent subpicosecond timescales of the associated charge carrier motion. In recent years, this mechanism has been employed as a versatile source for $\mathrm{THz}$ waveforms [3-5]. Because the radiation encodes key fingerprints of the ultrafast electron dynamics, terahertz emission spectroscopy (TES) represents a powerful means of probing photo-induced phenomena at surfaces or interfaces. A direct extraction of the generating current is possible by a quantitative analysis of the time-domain structure of the transients [6]. Thus, in far-field spectroscopy, surface dynamics in topological materials $[7,8]$ or the interlayer charge transfer in van der Waals heterostructures [9] have been investigated.

The spatial resolution in TES is generally unrelated to the macroscopic wavelengths in the $\mathrm{THz}$ spectral range, but rather set by the optical excitation $[10,11]$. Still, the approach lacks nanoscale spatial sensitivity. To overcome this challenge, TES has recently been merged with $\mathrm{THz}$ near-field microscopy [11-18] and termed terahertz emission nanoscopy (TEN) $[11,14]$ paving the way for investigations of nanostructures. While the near-field probe can efficiently confine the emerging long-wavelength radiation to its sharp apex and subsequently scatter it to the far field, the metallic tip also alters the temporal structure of the detected waveforms. Accurate predictions of the changes to the $\mathrm{THz}$ transients induced by the light scattering process have so far mostly remained elusive. This has hampered a link between the experimental waveforms and the ultrafast current that acts as the nanoscale source of the $\mathrm{THz}$ radiation. A quantitative description of TEN is, however, required to access vital information such as the influence of the local background doping, the topography, or the dielectric environment on the intrinsic ultrafast electron dynamics at the sample surface with subwavelength spatial resolution.

Conversely, light scattering from the tip-sample system can readily be described by state-of-the-art models $[19,20]$ in the midinfrared spectral range [21] where the probe geometry can often be simplified, for example, to a set of interacting dipoles or rotationally symmetric objects. This is in stark contrast to the actual experimental situation of $\mathrm{THz}$ nanoscopy [16-18], where the full dimensions of the tip and even the cantilever geometry affect the spectral response. Finite element method (FEM) simulations $[16,17,22]$ have proven to be an invaluable tool for obtaining nanoscale field distributions in the vicinity of realistic probes. Thereby, the scattering of $\mathrm{THz}$ electric fields from the tip-sample system can be described quantitatively and, in principle, without any free parameters. The scarcity of FEMbased predictions of the $\mathrm{THz}$ response of typical near-field probes in the literature arises from the challenge of implementing a macroscopic simulation volume that accommodates the long wavelengths of the $\mathrm{THz}$ radiation, while ensuring nanoscale resolution at the tip apex.

Here, we study the $\mathrm{THz}$ waveforms generated at the InAs surface by FEM simulations with a multiscale mesh. The ultrafast separation of charges underlying the photo-Dember effect is induced by the significantly different mobilities of photogenerated electrons and holes [6,23]. This ideal model system allows for a direct comparison between far-field TES and TEN. Hence, we identify the key aspects of the complicated probe geometry such as the cantilever length, which dictate the temporal structure of the near-field waveforms. Our simulations represent a widely applicable strategy for a quantitative modeling of TEN and should also aid in the design of optimized near-field probes. In our experiments (see Supplement 1, Section 1), we photoexcite an InAs surface with near-infrared pump pulses (center wavelength: $1030 \mathrm{~nm}$; pulse duration: 
$180 \mathrm{fs}$ ) generated by a low-noise thin-disk laser source [24]. This creates an ultrafast current near the sample surface [Fig. 1(a), inset] resulting in the emission of electromagnetic waves at $\mathrm{THz}$ frequencies. In TEN, this process is enhanced via evanescent coupling to the tip apex. The $\mathrm{THz}$ waveforms are subsequently recorded in the far field using electro-optic detection in both types of experiments. We rely on commercial probes typically employed in THz nanoscopy (e.g., 25PtIr200B-H40 by Rocky Mountain Nanotechnology LLC). Representative scanning electron microscopy images are depicted in Fig. 1(b). The probe features a conical tip with a radius of curvature of $40 \mathrm{~nm}$ at the sharp apex and a length of $\sim 80 \mu \mathrm{m}$. As we will demonstrate below, the complex geometry at the other end of the cantilever can be described by an effective length $L=325 \mu \mathrm{m}$ spanning the distance between the tip and the epoxy used to attach the cantilever to the support chip. Figures 1(c) and 1(d) illustrate the corresponding setting of the numerical simulations, which will be discussed later on. By removing the probe from the nearinfrared focal spot we can switch between TEN and TES. The emitted $\mathrm{THz}$ waveforms are collected by the off-axis parabolic mirror of a commercial near-field microscope and sampled electro-optically. For TEN, the electric fields are demodulated at the tip tapping frequency. The transients consist of a single optical cycle with a duration of $\sim 1$ ps [Fig. 2(a), top] corresponding to a spectrum centered near $\sim 1 \mathrm{THz}$ [Fig. 2(b), symbols]. There are several key differences between the waveform obtained with far-field spectroscopy (orange) and with $\mathrm{THz}$ nanoscopy (blue): First, the main oscillation cycle of the near-field waveform is slightly delayed and exhibits a shift in the carrier-envelope phase. Second, after $\sim 3$ ps, a replica waveform with flipped field polarity is detected in the tip-enhanced emission process manifesting as periodic, Fabry-Perot-like modulations of the spectral amplitudes [Fig. 2(b), blue].

To understand these findings, we perform FEM simulations of the emission of $\mathrm{THz}$ fields (see Supplement 1, Section 2). By calculating the response functions of the near-field probes, a theoretical comparison between TES and TEN is thus possible. Figure 1(c) depicts the entire simulation geometry that is surrounded by perfectly matched layers to suppress artefacts due to unphysical reflections at the simulation boundaries. The out-of-plane current $j_{z}$ [compare inset in Fig. 1(a)] induced by the photo-Dember effect at the InAs surface is modeled by a point dipole source positioned directly beneath the tip apex [Fig. 1(d)]. To describe the ultrafast temporal evolution of $j_{z}$ [Fig. 2(c)], we weight the spectral emission amplitude according to the analytical model introduced in Ref. [23]. The free parameters are the background doping of the InAs sample and the charge carrier density upon photoexcitation. The near fields $E^{\mathrm{NF}}$ (black) at the tip apex are directly proportional to the temporal derivative of $j_{z}$. To predict the emitted $\mathrm{THz}$ waveforms from the spectral amplitude $\bar{E}^{\mathrm{NF}}$ [see Fig. 2(d)], the response functions of the tip $\Omega^{T}$ [Figs. 2(e) and 2(f)] and the electro-optic detector $\Omega^{D}$ [green, Fig. 2(d)] are taken into account. Whereas the latter can readily be calculated considering the optical properties of the $\mathrm{GaP}$ detection crystal and the geometry of the electro-optic sampling, the former is obtained with the FEM simulations introduced in Fig. 1. To calculate the tip response function $\Omega^{T}$, we discretize the relevant frequency range $v$ supported by $\bar{E}^{\mathrm{NF}}$ and $\Omega^{D}$ from 0.2 up to $3.5 \mathrm{THz}$ in steps of $50 \mathrm{GHz}$. Next, the far fields $E^{\text {far }}$ emerging from this source are predicted based on the field distributions, employing

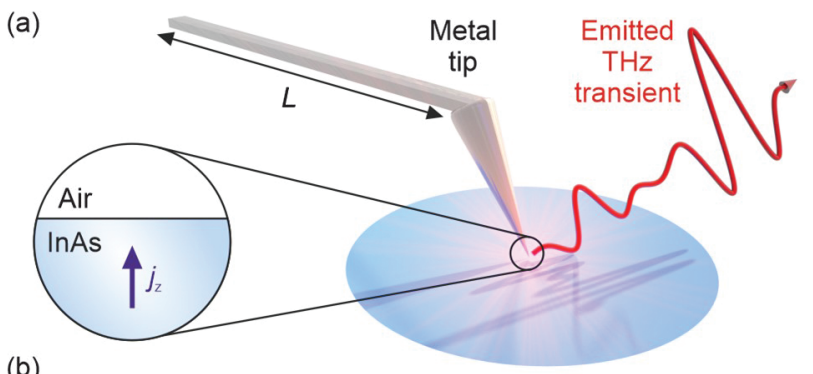

(b)
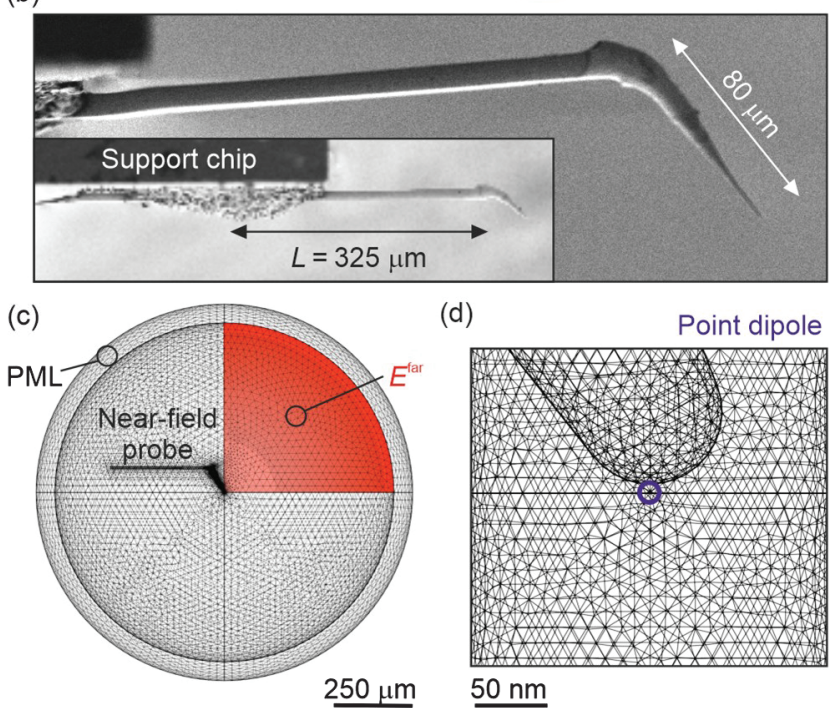

(d)

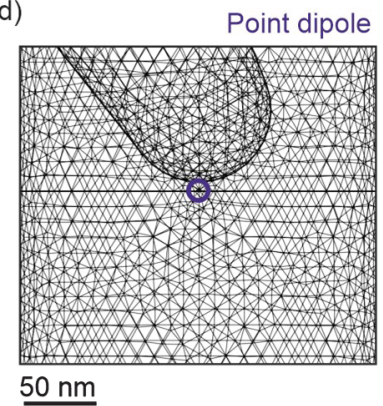

Fig. 1. Concept and simulation of $\mathrm{THz}$ emission nanoscopy. (a) Photoexcitation of an InAs surface leads to an out-of-plane current $j_{z}$ (inset) owing to the photo-Dember effect. The emerging $\mathrm{THz}$ radiation is coupled out by the sharp apex of a metal tip attached to a cantilever with characteristic length $L$. (b) Scanning electron microscopy images of typical near-field probes highlighting the relevant parameters. (c) Simulation geometry based on the finite element method surrounded by perfectly matched layers (PMLs). The farfield radiation $E^{\text {far }}$ is evaluated on the surface of the red-shaded area. (d) Close-up view of the tip above the sample including the point dipole (purple) acting as a source for the THz fields.

the Stratton-Chu formalism. Here, only a quarter of the simulation geometry [red area in Fig. 1(c)] is evaluated to emulate the collection of the radiation with the parabolic mirror.

The ratio between the vertically polarized far fields emitted in the simulations including the tip and cantilever, $E_{\mathrm{tip}}^{\mathrm{far}}$, and the fields emerging from the bare dipole itself, $E_{\text {bare }}^{\text {far }}$, serves to capture the light scattering from the probe in a complex response function:

$$
\Omega^{T}(v)=\frac{E_{\mathrm{tip}}^{\mathrm{far}}(v)}{E_{\mathrm{bare}}^{\mathrm{far}}(v)} .
$$

The probe features a broadband response $\Omega^{T}$, whose amplitude spectrum [Fig. 2(e)] is highly structured due to periodic enhancements at equidistant frequencies that are accompanied by characteristic oscillations in the phase [Fig. 2(f)]. In addition, the tip effectively acts as a low-pass filter as reported previously [25]. Interestingly, when fully neglecting the cantilever (black), $\Omega^{T}$ instead exhibits the characteristics of a single antenna resonance of the bare tip $[16,17]$. The spectra of the emerging THz radiation in TES and TEN are determined by 
a multiplication of $\bar{E}^{\mathrm{NF}}$ with the respective response functions in Fourier space. We obtain excellent agreement between the experiments [Fig. 2(b), symbols] and the simulations (lines). In the time domain [Fig. 2(a)], the simulated near-field waveform is more asymmetric, its maximum is delayed by $\sim 130 \mathrm{fs}$, and the transient contains trailing oscillations, whose timing is accurately predicted by the simulation geometry, specifically the cantilever length $L$. Experimentally, an absolute comparison of the delay between the main oscillation cycles of the TES and TEN waveforms is challenging due to potential, subtle beam path differences that might affect the relative timing.

Next, we elaborate on the geometrical origin of the intricate spectral shape of the tip response function $\Omega^{T}$. To this end, we study the field distributions of the out-of-plane electric field
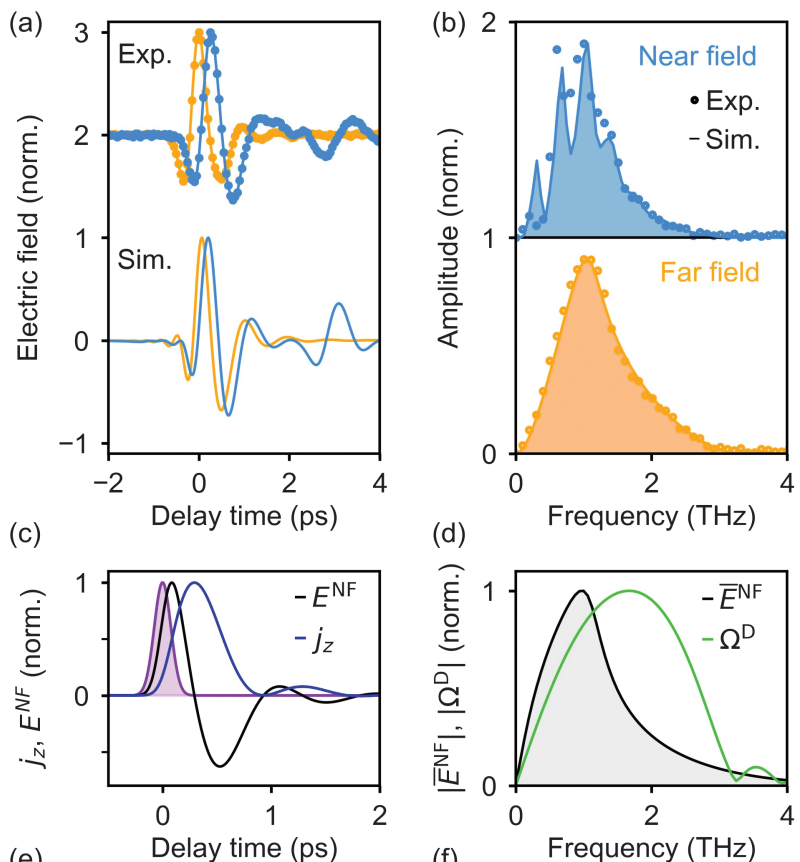

(e)

(d)
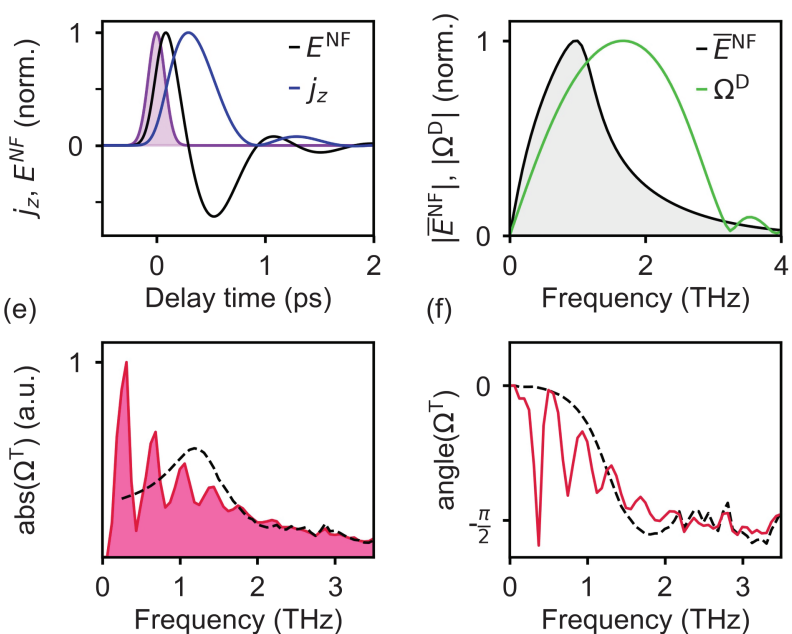

(a)

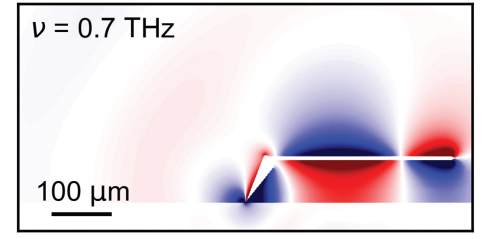

$v=1.05 \mathrm{THz}$

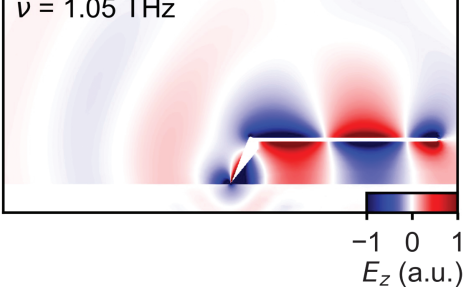

(b)

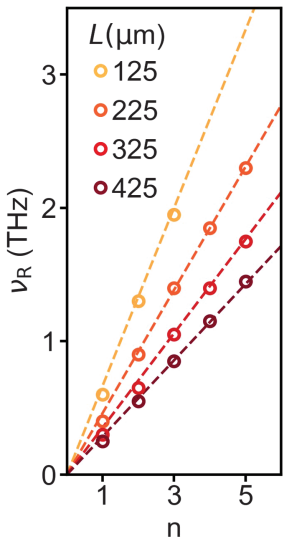

Fig. 3. Geometrical origin of the spectrally structured tip response. (a) Maps of the out-of-plane electric field $E_{z}$ for $L=325 \mu \mathrm{m}$ and different frequencies $v$. (b) Resonance frequencies $v_{R}$ of order $n$ for different cantilever lengths $L$. The dashed lines represent fits with Eq. (2).

$E_{z}$ in the vicinity of the probe. The maps in Fig. 3(a) reveal that the coupling to the far field is enhanced for frequencies $v_{R}$ corresponding to resonances of order $\mathrm{n}$. As the length $L$ of the cantilever is reduced, the resonances are blueshifted in energy [Fig. 3(b)]. We can clearly resolve the spectral enhancements up to order $n=5$ (corresponding to frequencies up to $\sim 2 \mathrm{THz}$ ) before they are obscured by the broadband scattering response of the tip [compare Fig. 2(e)]. For a wide range of cantilever lengths, the resonances $v_{R}$ can perfectly be described by a Fabry-Perot-like interference:

$$
v_{R}=\frac{c \cdot n}{2 \cdot(l+L)} .
$$

Here, $c$ is the speed of light, and $l$ is the effective length of the tip determined by fitting the data [lines in Fig. 3(b)]. The obtained value of $l=100 \mu \mathrm{m}$ is slightly larger than the nominal height of $\sim 80 \mu \mathrm{m}$ [Fig. 1(b)] most likely due to the tilted cone shape of the tip, which increases the optical path. In general, the dielectric response of the sample has only a minor impact on $\Omega^{T}$, confirming that the spectral response is mostly governed by the probe geometry. In the phase response, the resonance condition in Eq. (2) manifests as periodic oscillations [compare Fig. 2(f)] formed by the superposition of the highly dispersive cantilever resonances, whose oscillator strength is diminished for increasing resonance frequency. For frequencies beyond $\sim 2 \mathrm{THz}$, the relative phase approaches a nearly constant value of $-\frac{\pi}{2}$ set by the antenna resonance of the bare tip [Fig. 2(f)]. These features give rise to the time delay of the near-field waveform, its shift in carrier-envelope phase as well as the additional trailing oscillations.

A variation of the cantilever length in Fig. 4(a) reveals that the replica pulse can be identified as a reflection of the main pulse at the end of the cantilever. As a result, the trailing oscillations occur after a delay with respect to the main oscillation cycle (which originates from beneath the tip apex) that is dictated by the path difference of $\sim 2 \cdot(l+L)$ set by the tip and cantilever dimensions (oblique dashed line). In contrast, the magnitude of the peak electric field and the temporal structure (vertical dashed line) of the main pulse is basically unaffected by the changes in $L$. In the frequency domain, the timing of the replica pulse

the response of the tip excluding the cantilever $(L=0 \mu \mathrm{m})$. All other simulations were performed with cantilever length $L=325 \mu \mathrm{m}$. 

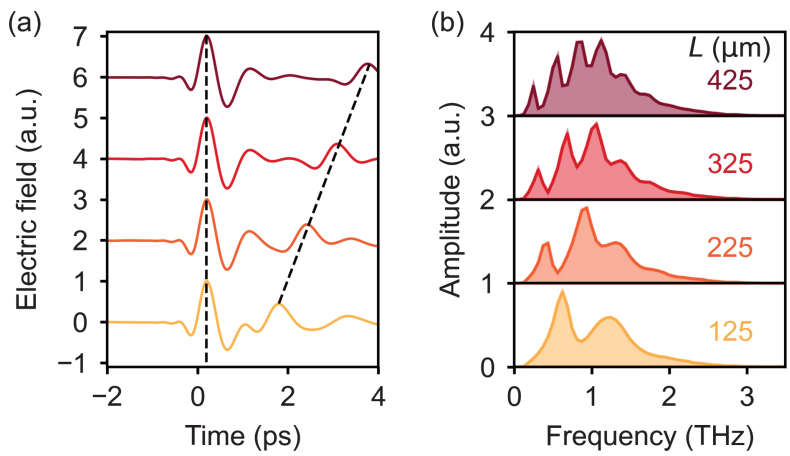

Fig. 4. Impact of the cantilever length. (a) Simulated waveforms obtained in analogy to Fig. 2 for different cantilever lengths $L$. The dashed lines indicate that the main oscillation cycle is hardly affected (vertical line), whereas the timing of the replica pulse is dictated by $L$ (oblique line). (b) Spectral amplitudes of the waveforms in (a).

determines the periodical modulations of the spectral amplitude of the THz waveforms [Fig. 4(b)]. For $L=115 \mu \mathrm{m}$, only two resonances lie within the relevant spectral range determined by the bandwidth of $j_{z}$.

The precise knowledge of the tip-enhanced $\mathrm{THz}$ emission presented in Figs. 2 and 4 sets the stage for reconstructing the underlying ultrafast current $j_{z}$ even with rather complex temporal structures of the detected waveform. Hence, in future studies an ideal tip and cantilever geometry design that also maximizes the electric field strength at the electro-optic detector can be pursued. In essence, the goal is to optimize the magnitude, shape, and phase of $\Omega^{T}$ within the spectral window set by $\bar{E}^{\mathrm{NF}}$ and $\Omega^{D}$ for each specific experiment, for example, by tailoring the angle between the tip and cantilever or the geometry of the cone-shaped tip [26].

In conclusion, the key differences between far-field and near-field $\mathrm{THz}$ emission waveforms emerging from photoexcited InAs surfaces are a more asymmetric and delayed main oscillation cycle in the latter case. These features are quantitatively reproduced by finite element simulations based on a realistic tip and cantilever geometry. The additional trailing oscillations in the nanoscopy experiments were unambiguously identified as reflections of the emerging radiation off the end of the cantilever. In the frequency domain, this phenomenon manifests as multiple, Fabry-Perot-like interferences within the relevant spectral range set by the ultrafast generating current. Our parameter-free modeling of the response function of the tip-sample system provides solid ground for tailoring probe geometries with optimized performance for terahertz emission nanoscopy. Furthermore, replacing the point dipole source with an external illuminating $\mathrm{THz}$ electric field, enables an adaption of these quantitative simulations for subcycle nearfield microscopy. Hence, reliable scattering models for $\mathrm{THz}$ nanospectroscopy can be developed in the future.

Funding. Deutsche Forschungsgemeinschaft (Project-ID 314695032, SFB 1277 (Subproject A05)); Alexander von Humboldt-Stiftung; Defense Advanced Research Projects Agency (D18AC00014).

Acknowledgment. The authors thank Imke Gronwald for assistance with the SEM. F. M. acknowledges financial support by the Alexander von Humboldt Foundation. Research at Columbia is supported by the DARPA DRINQS Program.

Disclosures. The authors declare no conflict of interest.
Data Availability. Data underlying the results presented in this paper are not publicly available at this time but may be obtained from the authors upon reasonable request.

Supplemental document. See Supplement 1 for supporting content.

\section{REFERENCES}

1. P. U. Jepsen, D. G. Cooke, and M. Koch, Laser Photon. Rev. 5, 124 (2011).

2. R. Ulbricht, E. Hendry, J. Shan, T. F. Heinz, and M. Bonn, Rev. Mod. Phys. 83, 543 (2011).

3. A. Leitenstorfer, S. Hunsche, J. Shah, M. C. Nuss, and W. H. Knox, Phys. Rev. Lett. 82, 5140 (1999).

4. T. Kampfrath, M. Battiato, P. Maldonado, G. Eilers, J. Nötzold, S. Mährlein, V. Zbarsky, F. Freimuth, Y. Mokrousov, S. Blügel, M. Wolf, I. Radu, P. M. Oppeneer, and M. Münzenberg, Nat. Nanotechnol. 8, 256 (2013).

5. R. A. Lewis, J. Phys. D 47, 374001 (2014).

6. M. B. Johnston, D. M. Whittaker, A. Corchia, A. G. Davies, and E. H. Linfield, Phys. Rev. B 65, 165301 (2002).

7. L. Braun, G. Mussler, A. Hruban, M. Konczykowski, T. Schumann, M. Wolf, M. Münzenberg, L. Perfetti, and T. Kampfrath, Nat. Commun. 7, 13259 (2016).

8. Y. Gao, S. Kaushik, E. J. Philip, Z. Li, Y. Qin, Y. P. Liu, W. L. Zhang, Y. L. Su, X. Chen, H. Weng, D. E. Kharzeev, M. K. Liu, and J. Qi, Nat. Commun. 11, 720 (2020).

9. E. Y. Ma, B. Guzelturk, G. Li, L. Cao, Z.-X. Shen, A. M. Lindenberg, and T. F. Heinz, Sci. Adv. 5, eaau0073 (2019).

10. T. Kiwa, M. Tonouchi, M. Yamashita, and K. Kawase, Opt. Lett. 28, 2058 (2003).

11. P. Klarskov, H. Kim, V. L. Colvin, and D. M. Mittleman, ACS Photon. 4, 2676 (2017).

12. H. T. Stinson, A. Sternbach, O. Najera, R. Jing, A. S. Mcleod, T. V. Slusar, A. Mueller, L. Anderegg, H. T. Kim, M. Rozenberg, and D. N. Basov, Nat. Commun. 9, 3604 (2018).

13. J. Zhang, X. Chen, S. Mills, T. Ciavatti, Z. Yao, R. Mescall, H. Hu, V. Semenenko, Z. Fei, H. Li, V. Perebeinos, H. Tao, Q. Dai, X. Du, and M. Liu, ACS Photon. 5, 2645 (2018).

14. Z. Yao, V. Semenenko, J. Zhang, S. Mills, X. Zhao, X. Chen, H. Hu, R. Mescall, T. Ciavatti, S. March, S. R. Bank, T. H. Tao, X. Zhang, V. Perebeinos, Q. Dai, X. Du, and M. Liu, Opt. Express 27, 13611 (2019).

15. A. Pizzuto, D. M. Mittleman, and P. Klarskov, Opt. Express 28, 18778 (2020).

16. S. Mastel, M. B. Lundeberg, P. Alonso-González, Y. Gao, K. Watanabe, T. Taniguchi, J. Hone, F. H. L. Koppens, A. Y. Nikitin, and R. Hillenbrand, Nano Lett. 17, 6526 (2017).

17. T. Siday, L. L. Hale, R. I. Hermans, and O. Mitrofanov, ACS Photon. 7, 596 (2020).

18. M. Plankl, P. E. Faria Junior, F. Mooshammer, T. Siday, M. Zizlsperger, F. Sandner, F. Schiegl, S. Maier, M. A. Huber, M. Gmitra, J. Fabian, J. L. Boland, T. L. Cocker, and R. Huber, "Subcycle contactfree nanoscopy of ultrafast interlayer transport in atomically thin heterostructures," doi: 10.1038/s41566-021-00813-y.

19. A. S. McLeod, P. Kelly, M. D. Goldflam, Z. Gainsforth, A. J. Westphal, G. Dominguez, M. H. Thiemens, M. M. Fogler, and D. N. Basov, Phys. Rev. B 90, 085136 (2014).

20. B. Hauer, A. P. Engelhardt, and T. Taubner, Opt. Express 20, 13173 (2012).

21. X. Chen, D. Hu, R. Mescall, G. You, D. N. Basov, Q. Dai, and M. Liu, Adv. Mater. 31, 1804774 (2019).

22. F. Mooshammer, M. A. Huber, F. Sandner, M. Plankl, M. Zizlsperger, and R. Huber, ACS Photon. 7, 344 (2020).

23. A. Reklaitis, J. Appl. Phys. 108, 053102 (2010).

24. O. Pronin, J. Brons, C. Grasse, V. Pervak, G. Boehm, M.-C. Amann, V. L. Kalashnikov, A. Apolonski, and F. Krausz, Opt. Lett. 36, 4746 (2011).

25. K. Wang, D. M. Mittleman, N. C. J. van der Valk, and P. C. M. Planken, Appl. Phys. Lett. 85, 2715 (2004).

26. S. Thomas, G. Wachter, C. Lemell, J. Burgdörfer, and P. Hommelhoff, New J. Phys. 17, 063010 (2015). 